This item was submitted to Loughborough's Research Repository by the author.

Items in Figshare are protected by copyright, with all rights reserved, unless otherwise indicated.

\title{
Magnetic susceptibility and spin dynamics of a polyoxovanadate cluster: A proton NMR study of a model spin tetramer
}

\section{PLEASE CITE THE PUBLISHED VERSION}

https://doi.org/10.1103/PhysRevB.69.094436

\section{PUBLISHER}

(c) American Physical Society (APS)

\section{VERSION}

AM (Accepted Manuscript)

\section{PUBLISHER STATEMENT}

This work is made available according to the conditions of the Creative Commons Attribution-NonCommercialNoDerivatives 4.0 International (CC BY-NC-ND 4.0) licence. Full details of this licence are available at: https://creativecommons.org/licenses/by-nc-nd/4.0/

\section{LICENCE}

CC BY-NC-ND 4.0

\section{REPOSITORY RECORD}

Procissi, D., A. Shastri, loannis Rousochatzakis, M. Al Rifai, P. Kogerler, Marshall Luban, B.J. Suh, and Ferdinando Borsa. 2019. "Magnetic Susceptibility and Spin Dynamics of a Polyoxovanadate Cluster: A Proton NMR Study of a Model Spin Tetramer". figshare. https://hdl.handle.net/2134/34421. 


\section{Magnetic susceptibility and spin dynamics of polyoxovanadate cluster $\{$ V12\}: a proton NMR study of a model spin tetramer}

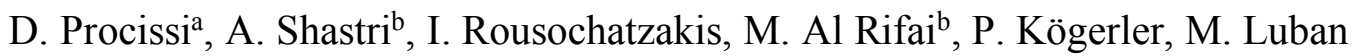

Department of Physics and Astronomy and Ames Laboratory, Iowa State University, Ames, Iowa, USA, 50011

B. J. Suh

Department of Physics, The Catholic University of Korea, Puchon, 420-743, Korea

F. Borsa

Department of Physics and Astronomy and Ames Laboratory, Iowa State University, Ames, Iowa, USA, 50011 and

Dip. Fisica "A. Volta" e Unita' INFM di PAvia, Via Bassi 6, Pavia. I27100, Italia

PACS: 76.60.-k, 76.60.Es, 76.60.Jx, 75.50.Xx, 75.30.Et, 75.30.Gw

\section{Abstract}

We report susceptibility and nuclear magnetic resonance (NMR) measurements in a polyoxovanadate compound with formula $(\mathrm{NHEt})_{3}\left[\mathrm{~V}^{\mathrm{IV}}{ }_{8} \mathrm{~V}_{4}{ }_{4} \mathrm{As}_{8} \mathrm{O}_{40}\left(\mathrm{H}_{2} \mathrm{O}\right)\right] \cdot \mathrm{H}_{2} \mathrm{O} \equiv\{\mathrm{V} 12\}$. The magnetic properties can be described by considering only the central square of localized $\mathrm{V}^{4+}$ ions and treated by an isotropic Heisenberg Hamiltonian of four intrinsic spins $1 / 2$ coupled by nearest-neighbor antiferromagnetic interaction with $J \sim 17.6 \mathrm{~K}$. In this

\footnotetext{
a procissi@ameslab.gov

b Permanent Address: Department of Physics and Astronomy, Minnesota State University-Moorhead, MN
} 
simplified description the ground state is nonmagnetic with $\mathrm{S}_{\mathrm{T}}=0$. The ${ }^{1} \mathrm{H}$ NMR line width (FWHM) data depends on both the magnetic field and temperature, and is explained by the dipolar interaction between proton nuclei and $\mathrm{V}^{4+}$ ion spins. The behavior of the nuclear spin-lattice relaxation rate $T_{1}^{-1}$ (NSLR) in the temperature range $(4.2-300 \mathrm{~K})$ is similar to that of $\chi T$ vs. $T$ and it does not show any peak at low temperatures contrary to previous observations in AFM rings with larger intrinsic spins. The results are explained by using the general features of the Moriya formula and by introducing a single $T$-independent broadening parameter for the electronic spin system. From the exponential $T$ dependence of $T_{I}{ }^{-1}$ at low $T(2.5 \mathrm{~K}<T<4.2 \mathrm{~K})$ we have obtained a field dependent gap following the linear relation $\Delta_{N M R}=\Delta_{0}-g \mu_{B} H$, with the gap $\Delta_{0} \sim 17.6 \mathrm{~K}$ in agreement with the susceptibility data. Below $2.5 \mathrm{~K}$ the proton $T_{1}^{-1}$ deviates from the exponential decrease indicating the presence of a small, almost temperature independent, but strongly field dependent, nuclear relaxation contribution, which we will investigate in detail in the near future.

\section{( I ) Introduction}

Magnetic polyoxovanadate clusters are a very interesting class of spin systems in which a few magnetic moments are strongly coupled by exchange interaction and are arranged in a vast variety of both geometrical and spin structures ${ }^{1}$. Within this class of molecules the $(\mathrm{NHEt})_{3}\left[\mathrm{~V}^{\mathrm{IV}}{ }_{8} \mathrm{~V}_{4} \mathrm{As}_{8} \mathrm{O}_{40}\left(\mathrm{H}_{2} \mathrm{O}\right)\right] \cdot \mathrm{H}_{2} \mathrm{O} \equiv\{\mathrm{V} 12\}$ cluster is comprised of 12 vanadium atoms arranged in a stack of three V squares as shown in Fig1(a). The top and bottom squares form strongly antiferromagnetically (AFM) coupled singlet states at room temperature and below and thus do not contribute significantly to the magnetic properties of the cluster at $T \leq 300 \mathrm{~K}$

2. The central square of $\mathrm{V}^{4+}$ ions, on the other hand, forms a square of $\mathrm{s}=1 / 2$ localized 
moments coupled by an almost isotropic AFM nearest-neighbor exchange interaction $J / k_{B}=17.6 K$. Thus $\{\mathrm{V} 12\}$ behaves as a prototype of a spin $1 / 2$ Heisenberg tetramer. The investigation of the spin dynamics of this molecule over the whole temperature range and for different values of the external magnetic field is of interest because one can follow the evolution from the high temperature regime of uncorrelated paramagnetic spins $\left(k_{\mathrm{B}} T>>J\right)$ to the low temperature regime with a ground state of total spin $S_{T}=0$. The nuclear magnetic resonance (NMR) of protons probes the spin dynamics of the system since the protons in the molecule are coupled to the $\mathrm{V}^{4+}$ electron spins via nuclear-electron dipolar interactions. In particular the proton spin-lattice relaxation rate $T_{1}^{-1}$ is proportional to the low frequency part of the spectral density of the electron spin fluctuations ${ }^{3}$. As pointed out in a preliminary report on $\{\mathrm{V} 12\}^{4}$, the low spin value $(\mathrm{s}=1 / 2)$ of each magnetic moment makes this system a good quantum counterpart of several other investigated AFM rings with high intrinsic spin value $(s=5 / 2)$, i.e. nearly classical spins ${ }^{5,6}$. Unfortunately, no "bonafide" AFM ring system, with $\mathrm{S}_{\mathrm{T}}=0$ ground state, composed of spins $\mathrm{s}=1 / 2$ is presently available except for $\mathrm{Cu} 8$ ring which, however, has an exchange interaction constant $(J)$ so large that the spin system is in the $\mathrm{S}_{\mathrm{T}}=0$ ground state at room temperature and below ${ }^{7}$. Thus V12 appears to be the only $s=1 / 2$ single quantum system whose results can be compared with the $s=1 / 2$ classical AFM rings.

In the present paper we report a detailed proton NMR investigation of the spin dynamics of the model spin tetramer aimed at testing the following issues: (i) the behavior of the electronic spin correlation function in the high temperature regime $\left(k_{B} T>J\right)$; (ii) the evolution of the spin correlations when the temperature becomes of the order of the exchange coupling $J\left(k_{B} T \sim J\right)$; (iii) the spin fluctuations at very low temperature when the 
molecular magnet is mostly in its singlet ground state $\left(k_{B} T<J\right)$. In Sec. (II) we describe the experimental details of the NMR measurements. In Sec. (III) we present the experimental results including magnetic susceptibility results which will guide us in the interpretation of the NMR data. In Sec. (IV) we analyze the data by using the general features of the Moriya formula and exact first-principles results based on the isotropic Heisenberg model. Finally, in Sec. (V) we give a summary and conclusions of the paper.

\section{(II) Experimental details}

Measurements of magnetization versus temperature were performed at $0.5 \mathrm{~T}$ using Quantum Design MPMS superconducting quantum interference device (SQUID) magnetometers. The NMR measurements were performed on polycrystalline powder samples synthesized as described in the Ref. [2], by using a standard Fourier transform (FT) pulse spectrometer. The proton NMR line was sufficiently narrow $(<70 \mathrm{kHz})$ to be irradiated by a single radio frequency (rf) pulse of duration between 2-4 $\mu \mathrm{sec}$. The proton nuclear spin-lattice relaxation rate $T_{1}^{-1}$ was measured by monitoring the recovery of the nuclear magnetization following a short sequence of saturating radio frequency pulses. The recovery of the nuclear magnetization was found to be exponential in most cases over more than one decade. Each molecule contains many non equivalent protons with different dipolar coupling to the four $\mathrm{V}^{4+}$ magnetic moments, namely $24 \times 5$ protons belonging to the $\mathrm{C}_{2} \mathrm{H}_{5}$ groups attached on the outside of the vanadium cluster and the remaining four belonging to the two water molecules. Thus the observation of an exponential recovery law implies the presence of a common spin temperature ${ }^{8,9}$. The common spin temperature is established if $T_{1} \gg T_{2}$ where $T_{2}$ is the spin-spin relaxation time. Under these circumstances the measured 
$T_{1}^{-1}$ is the weighted average of the relaxation rate for the different protons in the molecule. At high temperature and high magnetic field we observed non-exponential behavior due to the breakdown of the common spin temperature approximation and the $T_{1}^{-1}$ quantity was derived from the initial part of the nuclear magnetization recovery curve (i.e. tangent to the

origin). In this case also the measured $T_{1}^{-1}$ is a weighted average of the different relaxation rates ${ }^{5,6}$. The spin-lattice relaxation rate in the rotating frame was measured by using an initial $\pi / 2$ rf pulse immediately followed by a lock-in RF pulse of intensity $\mathrm{H}_{1} \sim 0.001 \mathrm{~T}$ and of variable duration $\tau$. The $T_{1 \rho^{-1}}$ parameter was obtained from monitoring the amplitude of the free precession decay as a function of the duration $\tau$ of the lock-in pulse ${ }^{9}$.

\section{(III) Experimental Results}

The results for the magnetic susceptibility $\chi$ measured in our $\{\mathrm{V} 12\}$ sample at $0.5 \mathrm{~T}$ are plotted in Fig. 2 as $\chi T$ vs. $T$. The rapid drop of $\chi T$ for $T \leq 100 \mathrm{~K}$ is indicative of the nonmagnetic ground state $\left(S_{T}=0\right)$. The inset in Fig. 2 shows $\chi$ vs. $T$. The proton NMR line was found to be a single symmetric line whose full width at half maximum (FWHM) is plotted in Fig. 3 as a function of temperature at two different external magnetic fields $(0.5 \mathrm{~T}$ and 4.7T). The low temperature limit (i.e. $\sim 50 \mathrm{kHz}$ ) of the low field line width $T$-dependence, can be ascribed almost entirely to the nuclear dipole-dipole interaction among the 124 protons in the molecule. The partial line narrowing from $50 \mathrm{kHz}$ to $28 \mathrm{kHz}$ occurring on increasing temperature is due to the averaging of the nuclear dipolar interaction by the onset of molecular hindered rotation of the $\mathrm{C}_{2} \mathrm{H}_{5}$ groups. This can be inferred by the fact that a similar line narrowing is commonly observed in the same temperature range as here in many compounds containing the same radical group ${ }^{8,9}$. From the comparison of the line width 
data for the two fields there appears to be only a slight inhomogeneous field dependent broadening. The inhomogeneous broadening is due to the relatively small dipolar coupling of the protons with the for $V^{4+}$ magnetic moments. The nuclear spin-lattice relaxation rate $T_{1}^{-1}$ is shown as a function of temperature in Fig. 4 for an external field of $0.5 \mathrm{~T}$ and $4.7 \mathrm{~T}$ respectively. In the temperature range $(4.2-300 \mathrm{~K})$ the behavior of $T_{1}^{-1}$ vs. $T$ is similar to the behavior $\chi T$ vs. $T$. It is of particular importance to note that there is no enhancement of $T_{l}^{-1}$ at temperatures close to $J / k_{B} \sim 17 \mathrm{~K}$, contrary to what has been reported in other AFM rings and clusters with intrinsic spins $\mathrm{s}>1 / 2^{5,6}$. The $T_{I}{ }^{-1}$ results in the low temperature range $(1.5-4.2 \mathrm{~K})$ are shown separately in Fig. 5(a) and 5(b). It is noted that below 4K the majority of the molecules are in the nonmagnetic ground state and therefore the $T_{I}^{-1}$ data in this low$T$ regime are of particular interest and will be analyzed separately. A detailed field dependent study of $T_{1}^{-1}$ was performed at $300 \mathrm{~K}$ and the results are shown in Fig. 6. We have included two points which refer to $T_{1 \rho}^{-1}$ measurements at 4.7T. In this case the relaxation rate in the rotating frame probes the spectral density at $\omega_{1}=\gamma_{N} H_{1}$ and thus the points have been plotted in the graph at the magnetic field in the rotating frame $H_{1}=\frac{\omega_{1}}{\gamma_{N}} \sim 0.001 T$. The strong field dependence of $T_{l}^{-1}$ indicates that the spectral density of the magnetic fluctuations is peaked at low frequency, a characteristic feature of low dimensional Heisenberg systems ${ }^{5,6,10}$.

\section{(IV) Analysis of experimental results}




\section{(i) Magnetic susceptibility}

The magnetic susceptibility data in Fig. 2 can be fitted well by a theoretical calculation based on the exact solution of the Heisenberg Hamiltonian. In Ref. [2] the starting Hamiltonian is an empirical anisotropic Heisenberg Hamiltonian with four exchange

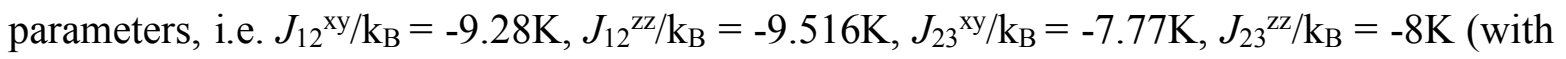
$J_{12}=J_{34}$ and $\left.J_{23}=J_{14}\right)^{\text {c }}$ as shown in the schematic depiction of Fig. 1(b). The choice of the Hamiltonian with these parameters was dictated by the need to reproduce the energy level scheme (shown in Fig. 7(a)) obtained directly from inelastic neutron scattering (INS) experiments ${ }^{2}$. However, an almost identical fit of the susceptibility can be obtained by using a far simpler isotropic Heisenberg Hamiltonian ${ }^{\mathrm{d}}$ with a single exchange parameter J. The eigenstates of $\mathrm{H}$ are of the form $\left|\mathrm{S}_{\mathrm{T}} \mathrm{M} \mathrm{S}_{13} \mathrm{~S}_{24}\right\rangle$, where $\overrightarrow{\mathrm{S}}_{13}=\overrightarrow{\mathrm{S}}_{1}+\overrightarrow{\mathrm{S}}_{3}, \overrightarrow{\mathrm{S}}_{24}=\overrightarrow{\mathrm{S}}_{2}+\overrightarrow{\mathrm{S}}_{4}$, $\overrightarrow{\mathrm{S}}_{T}=\overrightarrow{\mathrm{S}}_{13}+\overrightarrow{\mathrm{S}}_{24}$. Thus we have a singlet $\mathrm{S}_{\mathrm{T}}=0$ ground state $\left(\begin{array}{llll}0 & 0 & 1 & 1>\end{array}\right)$ with $E=0$, a triplet

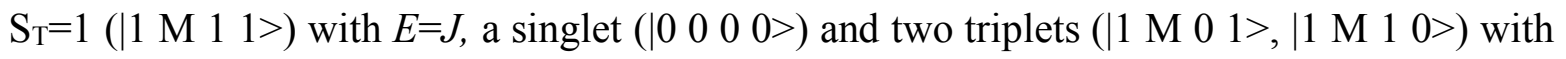
$\mathrm{E}=2 J$ and finally a quintet $\mathrm{S}_{\mathrm{T}}=2$ state $(\mid 2 \mathrm{M} 11>)$ with $E=3 J$, for a total number of $(2 s+1)^{N}=2^{4}=16$ states (see Fig. 7(b)). It is then straightforward to obtain the field-free partition function $Z^{11}$. Moreover the zero field molar susceptibility $\chi_{0}(T)$ is given by the fluctuation formula $\chi_{0}(T)=N_{A}\left[\left(g \mu_{B}\right)^{2} /\left(3 k_{B} T\right)\right]<S_{T^{2}}>$. One then finds

$$
\chi_{0} T=\frac{2 N_{A}\left(g \mu_{B}\right)^{2}}{Z k_{B}}\left(e^{-J / k_{B} T}+2 e^{-2 J / k_{B} T}+5 e^{-3 J / k_{B} T}\right),
$$

where $N_{A}$ is Avogadro's number. We found that Eq. (1) provides a very good fit to the experimental data upon choosing $g=1.97$ and $J / k_{B}=17.6 \mathrm{~K}$. The theoretical susceptibility

\footnotetext{
${ }^{\mathrm{c}}$ In Ref. 2 the convention $H=-2 \Sigma_{i j} J_{i j}^{\alpha \beta}\left(\mathrm{s}_{i}{ }_{\mathrm{S}} \mathrm{j}^{\beta}\right)$ was used ( $\alpha$ and $\beta$ take on the values $\left.\mathrm{x}, \mathrm{y}, \mathrm{z}\right)$.

${ }^{\mathrm{d}}$ Here we have used the convention $H=+J\left(\mathrm{~S}_{l} \cdot \mathrm{S}_{2}+\mathrm{S}_{2} \cdot \mathrm{S}_{3+} \mathrm{S}_{3} \cdot \mathrm{S}_{4}+\mathrm{S}_{4} \cdot \mathrm{S}_{I}\right)+2 J$.
} 
curve is given by the solid line in Fig. 2. This agreement is further evidence of the fact that the overall magnetic properties can be associated with the four central spins $\mathrm{s}=1 / 2$.

\section{(ii) ${ }^{1} \mathrm{H}$ NMR line width (FWHM) vs T}

The ${ }^{1} \mathrm{H}$ NMR line width $\Delta v$ (full width at half maximum FWHM) as a function of temperature and for two different fields is shown in Fig. 3. The dependence of $\Delta v$ on both the magnetic field and the temperature is ascribed to magnetic dipolar broadening via the dipolar interaction of the ${ }^{1} \mathrm{H}$ with the $\mathrm{V}^{4+}$ magnetic moments. For the dipolar magnetic broadening, the inhomogeneous line width $\Delta v$ for a given susceptibility per spin $\chi$, is given by $\frac{\Delta v}{v_{N}}=\frac{\Delta v}{\frac{\gamma_{N}}{2 \pi} H}=A_{z} \chi \sim \frac{\langle\mu\rangle}{r^{3} H}$, where $v_{N}$ is the proton Larmor frequency, $\mathrm{A}_{z}$ is the component of the dipolar hyperfine coupling constant along the direction of the external magnetic field $H$, and $\mathrm{r}$ is the average distance between the ${ }^{1} \mathrm{H}$ and the vanadium ions ${ }^{8}$. From the field dependence at room temperature (not shown here) and the susceptibility in Fig. 2 one can estimate the component $A_{z}$ of the dipolar coupling constant by considering the slope $\frac{\Delta v\left(H_{02}\right)-\Delta v\left(H_{01}\right)}{\frac{\gamma_{N}}{2 \pi}\left(H_{02}-H_{01}\right)}=A_{z} \chi \sim \frac{\langle\mu\rangle}{r^{3} H}$. We obtained a value of $A_{z} \sim 10^{22} \mathrm{~cm}^{-3}$ which

corresponds to the field generated by a $\mathrm{V}^{4+}$ magnetic moment at an average distance of about $3 \AA$ A. This agrees with the results for V15 and V6 given in Ref. [12]. The result demonstrates that ${ }^{1} \mathrm{H}$ NMR is a direct probe of the magnetic properties of the $\mathrm{V}$ ions in the $\{$ V12 $\}$ compound. 


\section{(iii) ${ }^{1} \mathrm{H}$ spin-lattice relaxation rate $T_{1}{ }^{-1}$}

One can obtain a general expression for the spin-lattice relaxation rate $T_{1}^{-1}$ through a method based on a perturbative treatment ("weak collision approach") of the dipolar coupling between nuclear and paramagnetic spins ${ }^{3,8,9}$. The major features of the spin-lattice relaxation rate can be easily seen in the following formula

$$
T_{1}^{-1}=\left(\gamma_{e} \gamma_{N} \hbar^{2}\right)^{2} \sum_{i j, a a^{\prime}} C_{i j}^{a a^{\prime}} \int_{-\infty}^{+\infty} d t e^{i \omega_{N} t}\left\langle S_{i}^{a}(0) S_{j}^{a^{\prime}}(t)\right\rangle
$$

where the coefficients $C_{i j}^{a a^{\prime}}$ contain all the detailed geometrical coefficients of the dipolar interactions, $\{i, j\}$ are paramagnetic spin sites and $\left\{a, a^{\prime}\right\}$ cartesian coordinates. It can be easily seen that the difficulty in evaluating $T_{1}^{-1}$ in (2) arises firstly from the calculation of $C_{i j}^{a a^{\prime}}$ and secondly from the calculation of all the spin-spin correlation functions. The following simplifications have been made in order to get the major features:

(1) We use the isotropic Heisenberg Hamiltonian ${ }^{\text {d }}$ that we used for the susceptibility;

(2) We replace the delta functions that arise from (2) by Lorentzian functions with a single temperature independent broadening parameter $\omega_{0}$ whose physical interpretation is in terms of a cut-off frequency of the spin-spin correlation functions due to couplings that don't commute with the Heisenberg isotropic exchange ${ }^{10}$;

(3) The difference in the dipolar interactions of the inequivalent protons (different $C_{i j}^{a a^{\prime}}$ for different protons) in the molecule is treated by averaging out the geometrical details of the system, i.e. we deal with a single, average $T_{1}^{-1}$; 
(4) Since the energies of the electronic spin system are of the order of $J / k_{B} \sim 17.6 K$, we keep only terms that correspond to zero transition frequencies. This simplification is valid for fields far below the first level crossing $(\sim 13.3 \mathrm{~T})$;

By using the total spin symmetries of the Hamiltonian, i.e. $\left[H, S_{T}^{2}\right]=\left[H, S_{T}^{z}\right]=0$ and the resulting selection rules, we get the following general form for the spin lattice relaxation rate $^{3,13}$

$$
T_{1}^{-1}=F_{L}(T, H) \frac{\omega_{0}}{\omega_{0}{ }^{2}+\omega_{N}{ }^{2}}+F_{T}(T, H) \frac{\omega_{0}}{\omega_{0}{ }^{2}+\omega_{e}{ }^{2}},
$$

where the first term comes from the (auto and pair) longitudinal correlation functions and the second comes from the transverse terms. The temperature and field dependence of $F_{L}, F_{T}$ arises from the various Boltzmann factors of the electronic spin levels. There is no need for a further simplification of setting $F_{L}$ and $F_{T}$ equal ${ }^{13}$.

In the expressions for $F_{L}, F_{T}$ in Eq. (3), the contribution from the ground state vanishes since the ground state is a non-degenerate $\mathrm{S}_{\mathrm{T}}=0$ state. We will use this additional feature in writing Eq. (4), and also in the low-T behavior of $T_{1}^{-1}$ (Eq. 6).

We are ready now to discuss our experimental results using the above general features of $T_{1}^{-1}$

\section{(a) $T_{1}^{-1}$ vs. field at $\boldsymbol{T}=\mathbf{3 0 0 K}$}

In the high temperature regime $(\mathrm{T}=300 \mathrm{~K})$ the Boltzmann factors in $F_{L}, F_{T}$ in (3) are all close to unity. Hence one should be able to reproduce the field dependence of $T_{1}^{-1}$ at $\mathrm{T}=300 \mathrm{~K}$ with the relation 


$$
T_{1}^{-1}=P \frac{\omega_{0}}{\omega_{0}{ }^{2}+\omega_{N}{ }^{2}}+Q \frac{\omega_{0}}{\omega_{0}{ }^{2}+\omega_{e}{ }^{2}},
$$

where $\mathrm{P}$ and $\mathrm{Q}$ are constants. Indeed, the field dependence of $T_{1}^{-1}$ at $\mathrm{T}=300 \mathrm{~K}$, can be well described by the relation

$$
T_{1}^{-1}=K \frac{H_{0}^{-1}}{1+\left(H / H_{0}\right)^{2}},
$$

where $K$ and $H_{0}$ are fitting parameters. From the fit in Fig. 6 one obtains $K / H_{0}=8.9 m \mathrm{sec}^{-1}$ and $H_{0}=1.3 T$. Eq. 4(b) arises from Eq. 4(a) in two limiting cases: (i) if $P<<Q$ and $\omega_{0} \sim \omega_{e}$ in which case one would have $\omega_{0}=\gamma_{e} H_{0} \sim 2.3 \times 10^{11} \mathrm{~Hz}$ and (ii) if $P \sim Q$ and $\omega_{0} \sim \omega_{N}<<\omega_{e}$ in which case one would have $\omega_{0}=\gamma_{N} H_{0} \sim 3.5 \times 10^{8} \mathrm{~Hz}$. Both choices are consistent with the fact that $T_{1 \rho}^{-1}$ value is of the order of the zero field extrapolated $T_{1}^{-1}$ since the spin-lattice relaxation in the rotating frame is proportional to the spectral density of the fluctuations at very low frequency, i.e. $\omega_{1} \sim 250 \mathrm{kHz}$. One could argue that there is no physical reason why $P<<Q$ since the constants are both related to the components of dipolar coupling tensor, and therefore the second choice is the relevant one. One way to determine this experimentally would be to perform a $T_{1}^{-1}$ measurement using a different (other than proton) nuclei. Unfortunately, an attempt to detect the ${ }^{51} \mathrm{~V}$ NMR signal was unsuccessful most likely due to the very short $T_{1}^{-1}$ and/or $T_{2}^{-1}$. Furthermore ${ }^{13} \mathrm{C}$ NMR in natural abundance yields a signal which is too weak for the required low field measurements.

(b) $T_{1}^{-1}$ vs. temperature at $\boldsymbol{H}=0.5 \mathrm{~T}$ for $\boldsymbol{T}>4.2 \mathrm{~K}$ 
For $\mathrm{H}=0.5 \mathrm{~T}$, we have $\hbar \omega_{e}<<k_{B} J$, and therefore we can neglect the field dependence in the Boltzmann factors in $F_{L}$ and $F_{T}$. Hence, the temperature dependence of $T_{1}^{-1}$ in Fig. 4 can be reproduced reasonably well by the following relation

$$
T_{1}^{-1}=\frac{1}{Z}\left(\alpha(H) \exp \left(-J / k_{B} T\right)+\beta(H) \exp \left(-2 J / k_{B} T\right)+\gamma(H) \exp \left(-3 J / k_{B} T\right)\right),
$$

where the field dependence in the fitting parameters $\alpha, \beta$ and $\gamma$ arises from the Lorentzian broadening as in equation (3). There is no contribution in (5) from the ground state, as indicated previously. The data of $T_{1}^{-1}$ for $H=0.5 \mathrm{~T}$ can be fitted by Eq. (5), for $T>4 \mathrm{~K}$, with the proper choice of fitting parameters $\alpha, \beta, \gamma$ (solid black curve in Fig. 4 with $\alpha=5.6$ $\left.\mathrm{msec}^{-1}, \beta=76.8 \mathrm{msec}^{-1}, \gamma=28 \mathrm{msec}^{-1}\right)$. One can see a deviation from the behavior given in Eq. (5) below $T=4.2 \mathrm{~K}$ in Fig. 4 which will be treated separately.

\section{(c) $T_{1}^{-1}$ vs. temperature at $\boldsymbol{H}=\mathbf{4 . 7 T}$ and for $\boldsymbol{T}>\mathbf{4 . 2 \mathrm { K }}$}

If one assumes that the constants $\alpha, \beta$ and $\gamma$ in Eq. (5) have the same field dependence as in Eq. 4(b) and that the parameter $\omega_{0}$, which defines the width of the Lorentzian function, is $T$-independent, one can try to fit the $T_{1}^{-1}$ data at $H=4.7 \mathrm{~T}(\mathrm{O})$ with the same set of parameters as for the fit at $0.5 \mathrm{~T}$ simply by rescaling by the field dependence given by Eq. $4(\mathrm{~b})$. We would expect that at $4.7 \mathrm{~T}$, which is approximately $1 / 3$ of the first level crossing field, the Boltzmann factors that enter $F_{L}, F_{T}$ would be affected significantly especially at low $T$. Indeed, there is a deviation below $10 \mathrm{~K}$ (see the dotted line in Fig. 4). It is noteworthy that one finds good qualitative agreement over a wide temperature range and this implies that the broadening parameter $\omega_{0}$ is $T$-independent at these temperatures. The above result suggest that the $T$ and $H$ dependence of $T_{1}^{-1}$ can be expressed in first approximation as the product 
of a temperature dependent function $f(T)$ and a field dependent function $g(H)$. This approximation obviously breaks down at high fields and low temperatures.

\section{(d) $T_{1}^{-1}$ vs. temperature for $\mathbf{T}<4.2 \mathrm{~K}$}

For temperatures below the ${ }^{4} \mathrm{He}$ boiling temperature only the ground state $(|0000\rangle)$ and the first excited state $(|1-111\rangle)$ are of importance. Then, for given field, the temperature dependence of $T_{1}^{-1}$, assuming again no $T$-dependence in $\omega_{0}$ in Eq. (3), follows the relation

$$
T_{1}^{-1} \sim \frac{A(H)}{Z} \exp \left(-\Delta_{N M R}(H) / k_{B} T\right)
$$

where $\Delta_{N M R}(H)=\Delta_{0}-\Delta E(H)$, with $\Delta_{0}=J$, is the gap between the ground state $S_{T}=0$ and $S_{T}=1$ excited state and $A(H)$ is a fitting parameter for each field coming from the Lorentzian broadening. One can easily see that $A(H) \exp \left(-\Delta_{N M R}(H) / k_{B} T\right)$ in Eq. (6) corresponds to $\alpha(H)$ in (5) when $k_{B} T>>$.

The initial part of the curve $(2.5 \mathrm{~K}<T<4.2 \mathrm{~K})$ in Fig. $5(\mathrm{a})$ and $5(\mathrm{~b})$ can indeed be fit with Eq.(6) (dotted curves in Fig. 5(a) and 5(b)) with $A(0.4 \mathrm{~T})=5.8 \mathrm{msec}^{-1}, A(1.34 \mathrm{~T})=1.3 \mathrm{msec}^{-1}$, $A(4.7 \mathrm{~T})=0.3 \mathrm{msec}^{-1}$, and $\Delta_{N M R}(0.4 \mathrm{~T}) / k_{B}=17.1 \mathrm{~K}, \Delta_{N M R}(1.34 \mathrm{~T}) / k_{B}=15.8 \mathrm{~K}$, and finally $\Delta_{N M R}(4.7 \mathrm{~T}) / k_{B}=11.4 \mathrm{~K}$. The obtained gap values $\Delta_{N M R}$ vs. $H$ are plotted in the inset of Fig. 5(b), the dashed curve represents the Zeeman field dependence of the gap given by $\Delta_{N M R}(H)=\Delta_{0}-g \mu_{B} H$, with $\Delta_{0} / k_{B}=17.6 \mathrm{~K}$.

For the case of the low field data $(0.4 \mathrm{~T}$ and $1.34 \mathrm{~T})$ one must note the deviation from the exponential thermally activated behavior given in Eq. 6 with $\Delta / / k_{B} \sim 17.6 \mathrm{~K}$ for $T<2.5 \mathrm{~K}$ (Fig. 5(a)), while in the case of the high field data (4.7T) the fit given by Eq. (6) reproduces 
the data also below $2.5 \mathrm{~K}$ as shown in Fig $5(\mathrm{~b})$. The weak temperature dependence below $2.5 \mathrm{~K}$ suggests that we fit the data in this regime by simply adding a T-independent but $\mathrm{H}$ dependent term in Eq. (6) (see Fig. 5). It is noted that in the octanuclear Cu8 antiferromagnetic ring, of $\mathrm{S}_{\mathrm{T}}=0$ ground state, a similar deviation at low temperatures was observed ${ }^{7}$. In $\mathrm{Cu} 8$ the ${ }^{63,65} \mathrm{Cu}$ NMR and NQR indicated the presence of non equivalent $\mathrm{Cu}$ sites suggesting a deviation from the exact isotropic Heisenberg Hamiltonian. Moreover, inelasting neutron scattering ${ }^{2}$ in the present system, indicated the presence of anisotropic exchange. However, no contribution to $T_{1}^{-1}$ can arise in Moriya's formula from a nondegenerate $\mathrm{S}_{\mathrm{T}}=0$ ground state, whatever the form of the Hamiltonian, and in particular whether $H$ includes anisotropic exchange and non-equivalent sites. Therefore we are lead to conclude that the very low-T contribution to $T_{1}^{-1}$ is coming either from paramagnetic "defects" or from a relaxation mechanism other than proton-vanadium dipolar coupling terms of Moriya's theory.

\section{(V) Summary and conclusions}

In this work we have presented comprehensive susceptibility and ${ }^{1} \mathrm{H}$ nuclear magnetic resonance experimental results of the polyoxovanadate cluster $\{\mathrm{V} 12\}$. The susceptibility experimental data were well fitted using results from exact calculations based on an isotropic Heisenberg Hamiltonian for the spin 1/2 tetramer. From the NMR spectral measurements and the temperature and field dependence of the NMR line width we have established that the inhomogeneous broadening is due to the dipolar coupling of the protons 
with the localized vanadium ions. The spin dynamics of the tetramer has been characterized through the proton spin-lattice relaxation rate, $T_{1}^{-1}$, in different temperature regimes and for different fields and we used general arguments (based on Moriya's first-principles treatment) to reproduce the results. At high temperature $\left(\mathrm{k}_{\mathrm{B}} \mathrm{T}>>\mathrm{J}\right) T_{1}^{-1}$ exhibits a strong and well defined field dependence which is well reproduced by a Lorentzian spectral density of the spin fluctuations. This field behavior is similar to that observed in one-dimensional magnetic systems ${ }^{10}$. In the intermediate temperature range $(10 \mathrm{~K}<T<300 \mathrm{~K})$ the temperature behavior of $T_{1}^{-1}$ is similar to the $T$-dependence of $\chi T$ indicating that the proton $T_{1}^{-1}$ is dominated by the amplitude of the local spin fluctuations. Particularly relevant is the absence of an enhancement of the relaxation rate $T_{1}^{-1}$ for $k_{B} T \sim J$ which is found in other similar molecular magnetic rings comprised of spins with $s>1 / 2{ }^{6}$. The fact that we were able to roughly reproduce the high and low field data simply by means of a rescaling factor is evidence of the fact that the $T$ and $H$ dependence of $T_{I}^{-1}$ can be expressed in first approximation as the product of two independent functions $f(T)$ and $g(H)$ and most importantly that the broadening parameter $\omega_{0}$ is weakly temperature dependent down to at least $10 \mathrm{~K}$. Finally, in the low $T$ regime $(2.5 \mathrm{~K}<\mathrm{T}<4.2 \mathrm{~K})$, and for both high and low fields, $T_{I}^{-1}$ decreases exponentially as the temperature is lowered. The value of the gap $\Delta$ obtained from the fit of the data is consistent with the simple linear field dependence $\Delta_{N M R}(H)=\Delta_{0}$ $g \mu_{B} H$ with $\Delta_{0}=17.6 \mathrm{~K}$ in excellent agreement with the susceptibility results and with INS and dynamic magnetization measurements ${ }^{14}$. For $T<2.5 \mathrm{~K}$ the temperature dependence of $T_{1}^{-1}$ deviates from the thermally activated exponential behavior and this is most evident in the low field data $(0.4 \mathrm{~T}$ and $1.34 \mathrm{~T})$. This deviation will be explored in detail in the near future. 
The most remarkable conclusion of the present work is found by comparing the behavior of the present $s=1 / 2$ tetramer with the $s>1 / 2$ AFM rings. Although in both cases the proton $T_{1}^{-1}$ can be well described by Moriya's theory, the characteristic frequency $\omega_{0}$ defining the broadening of the magnetic levels of the molecule and thus the spin fluctuations is quite different for the $\mathrm{s}=1 / 2$ quantum case and the $\mathrm{s}>1 / 2$ classical case. In $\mathrm{V} 12, \omega_{0}$ appears to be almost T-independent and thus it does not give rise to the peak in $T_{1}^{-1}$ observed in AFM rings ${ }^{4,6}$ where $\omega_{0}$ is strongly $\mathrm{T}$-dependent becoming of the order of the nuclear Larmor frequency at the peak ${ }^{15}$. Another important finding is the existence of a residual nuclear relaxation mechanism at very low temperatures. It will be noted that similar results were obtained in half-integer isotropic high-spin ground state molecules of different spin values ${ }^{16}$.

\section{Acknowledgements}

We would like to acknowledge fruitful discussion with H. Nojiri. Ames Laboratory is operated for the U.S. Department of Energy by Iowa State University under Contract No. W-7405-Eng-82.

\section{References}

${ }^{1}$ A. Müller, R. Sessoli, E. Krickemeyer, H. Bögge, J. Meyer, D. Gatteschi, L. Pardi, J.

Westphal, K. Hovemeier, R. Rohlfing, and J. Döring, Inorg. Chem. 36, 5239 (1997).

${ }^{2}$ R. Basler, G. Chaboussant, A. Sieber, A. Andres, M. Murrie, P. Kögerler, H. Bögge, D. C.

Crans, E. Krickemeyer, S. Janssen, H. Mutka, A. Müller, and H. Güdel, Inorg. Chem. 41, 5675-5685 (2002).

3 T. Moriya, Prog. Theor. Physics, 28, 512 (1962). 
4 B. J. Suh, D. Procissi, P. Kögerler, E. Micotti, A. Lascialfari, and F. Borsa, ICM, Rome, (2003).

5 A. Lascialfari, Z. H. Jang, F. Borsa, D. Gatteschi, and A. Cornia, J. Appl. Phys, 83, 6946 (1998).

6 A. Lascialfari, D. Gatteschi, F. Borsa, and A. Cornia, Phys. Rev. B, 55, 14341 (1997).

${ }^{7}$ A. Lascialfari, Z. H. Jang, F. Borsa, D. Gatteschi, A. Cornia, D. Rovai, A. Caneschi, P. Carretta, Phys. Rev B, 61, 6839, (2000)

8 A. Abragam, Principles of Nuclear Magnetism (Clarendon Press, Oxford, 1961)

${ }^{9}$ C. P. Slichter, Principles of Magnetic Resonance (Springer Verlag, Berlin, 1989)

${ }^{10}$ F. Borsa and M. Mali, Phys. Rev. B, 9, 2215 (1974).

11 The partition function is given by $Z=1+3 \exp \left(-J / k_{B} T\right)+7 \exp \left(-2 J / k_{B} T\right)+5 \exp \left(-3 J / k_{B} T\right)$

${ }^{12}$ D. Procissi, B. J. Suh, K.J. Jung, P. Kogerler, R. Vincent and F. Borsa, J. Appl. Phys. 93, $7810(2003)$

${ }^{13}$ M. Luban, F. Borsa, S. Bud'ko, P. Canfield, S. Jun, J. K. Jung, P. Kögerler, D. Mentrup, A. Müller, R. Modler, D. Procissi, B. J. Suh, M. Torikachvili, Phys. Rev. B, 66, 054407, (2002)

${ }^{14}$ H. Nojiri and M. Luban, (private communications), (2003).

${ }^{15}$ S. H. Baek, M. Luban, A. Lascialfari, E. Micotti, Y. Furukawa, F. Borsa, J. van Slageren, and A. Cornia (Unpublished)

${ }^{16}$ Z. Salman, A. Karen, P. Mendels, V. Marvaud, A. Scuiller, M.Verdaguer, J. S. Lord and C. Baines, Phys. Rev. B, 65, 132403 (2002) 


\section{Figures}

\section{Fig 1}

(a) Schematic representation of the $\left[\mathrm{V}^{\mathrm{IV}}{ }_{8} \mathrm{~V}_{4} \mathrm{As}_{8} \mathrm{O}_{40}\left(\mathrm{H}_{2} \mathrm{O}\right)\right]^{4-}$ cluster anion. The three planes formed by the vanadium ions (black spheres) are depicted in dark gray. (b) Schematic view of the central tetramer with definition of the superexchange and parameter pathways through the diarsenite ligands: (!) As; $(\mathrm{O}) \mathrm{O}^{1,2}$

\section{Fig 2}

Temperature dependence of $\chi T$ in $\{\mathrm{V} 12\}$ at $0.5 \mathrm{~T}$. The solid curve is the theoretical result (Eq.(1)) with $g=1.97$ and $J=17.6 \mathrm{~K}$. Also shown in the inset is the susceptibility $\chi$ vs. $T$ at $0.5 \mathrm{~T}$.

\section{Fig 3}

Temperature dependence of the proton line width (FWHM) in $\{$ V12 $\}$ at two different external fields $(\mathrm{O}) H_{o}=0.5 \mathrm{~T} ;$ (,) $H_{o}=4.7 \mathrm{~T}$.

\section{Fig 4}

Temperature dependence of the proton spin-lattice relaxation rate in $\{\mathrm{V} 12\}$ : (,) data at $H=0.5 \mathrm{~T}$. The solid curve is the best fit according to Eq. (5), with $\alpha=5.6 \mathrm{msec}^{-1}, \beta=76.8$ $\mathrm{msec}^{-1}, \gamma=28 \mathrm{msec}^{-1} ;(\mathrm{O})$ data at $4.7 \mathrm{~T}$. The dotted curve is the same curve as for the $0.5 \mathrm{~T}$ 
data but with a rescaling factor which takes into account the field dependence in Eq. 4(b) with $H_{0}=1.3 \mathrm{~T}$.

\section{Fig 5}

( a ) Semilog plot of $T_{1}^{-1}$ vs. $1000 / T$ for the low- $T$ range $(1.5 \mathrm{~K}-4.2 \mathrm{~K})$ for different magnetic fields: (O) 0.4T; (,) $1.34 \mathrm{~T}$ data. The dotted curves are fits according to Eq. (6) with $\mathrm{Z}=1$, $A(0.4 \mathrm{~T})=7 \mathrm{msec}^{-1}, A(1.34 \mathrm{~T})=1.3 \mathrm{msec}^{-1}$ and with $\Delta_{N M R}(0.4 T)=17.1 \mathrm{~K}, \Delta_{N M R}(1.34 T)=15.8 \mathrm{~K}$. The solid curve corresponds to the addition in Eq. (6) of a constant, field-dependent term $G(H)$, with $\mathrm{G}(0.4 \mathrm{~T})=3.9 \times 10^{-3} \mathrm{msec}^{-1}, \mathrm{G}(1.34 \mathrm{~T})=7.3 \times 10^{-4} \mathrm{msec}^{-1}$.

(b) (8) $4.7 \mathrm{~T}$ data. The dotted curve corresponds to Eq. (6) with $\mathrm{Z}=1, A(4.7 \mathrm{~T})=0.3 \mathrm{msec}^{-1}$ and $\Delta_{N M R}(4.7 T)=11.3 \mathrm{~K}$. The solid curve corresponds to the addition in Eq. (6) of the constant $G(H)$, with $\mathrm{G}(4.7 \mathrm{~T})=1.7 \times 10^{-4} \mathrm{msec}^{-1}$. The inset shows the obtained values of $\Delta_{N M R}$ vs. $H$, and the solid black line is the linear dependent expected behavior $\Delta_{N M R}(H)=\Delta_{0}-g \mu_{B}$ $H_{0}$ with $\Delta_{0}=17.6 \mathrm{~K}$ and $g \mu_{B}=1.33 \mathrm{~K} / \mathrm{T}$.

\section{Fig 6}

$T_{1}^{-1}$ vs. $H$ at $T=300 \mathrm{~K}$. The solid black curve represents the best fit to the data according to Eq. 4(b) with $H_{0}=1.3 \mathrm{~T}$ and $K / H_{0}=8.9 \mathrm{msec}^{-1}$. The two points plotted at very low fields are $T_{1 \rho}^{-1}$ measurements at $4.7 \mathrm{~T}$ (see text).

\section{Fig 7}

(a) Level scheme as obtained from INS ${ }^{2}$ (depicted are the observed inelastic neutron scattering transitions). 
(b) Level scheme as obtained from the isotropic Heisenberg model for four spin $\mathrm{s}=1 / 2$.
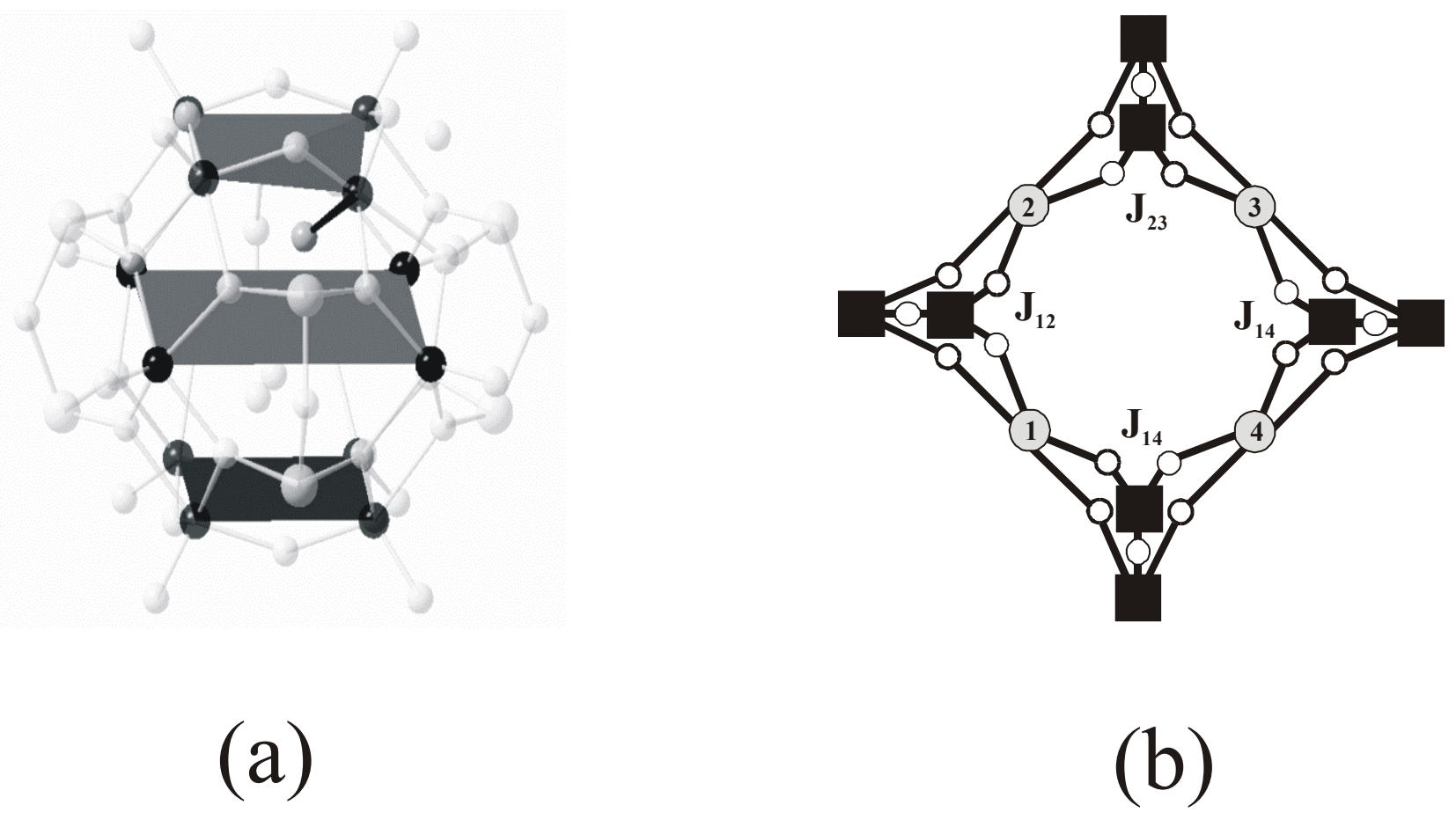

Fig 1 Procissi et al, Phys Rev B 


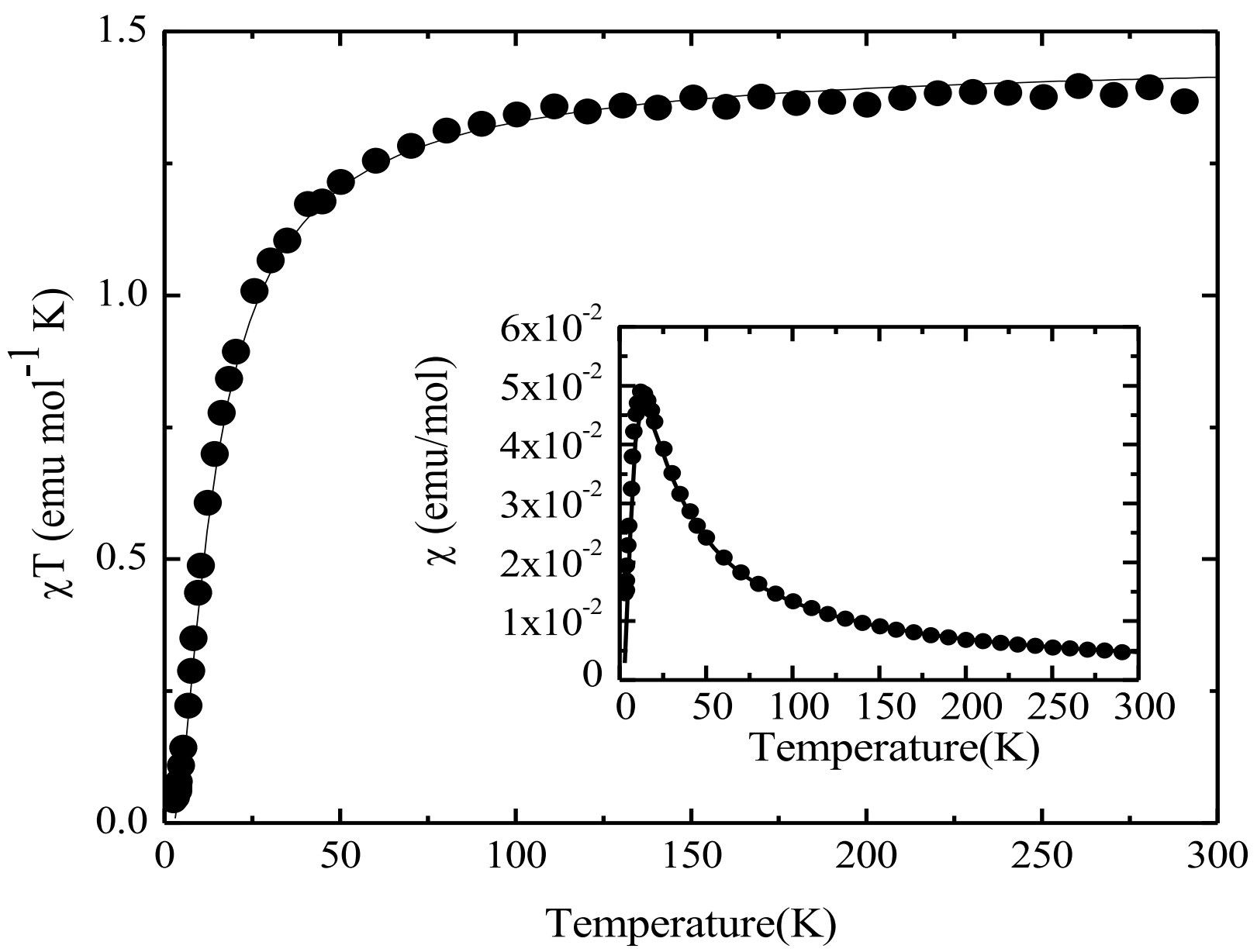

Fig 2 Procissi et al Phys Rev B 


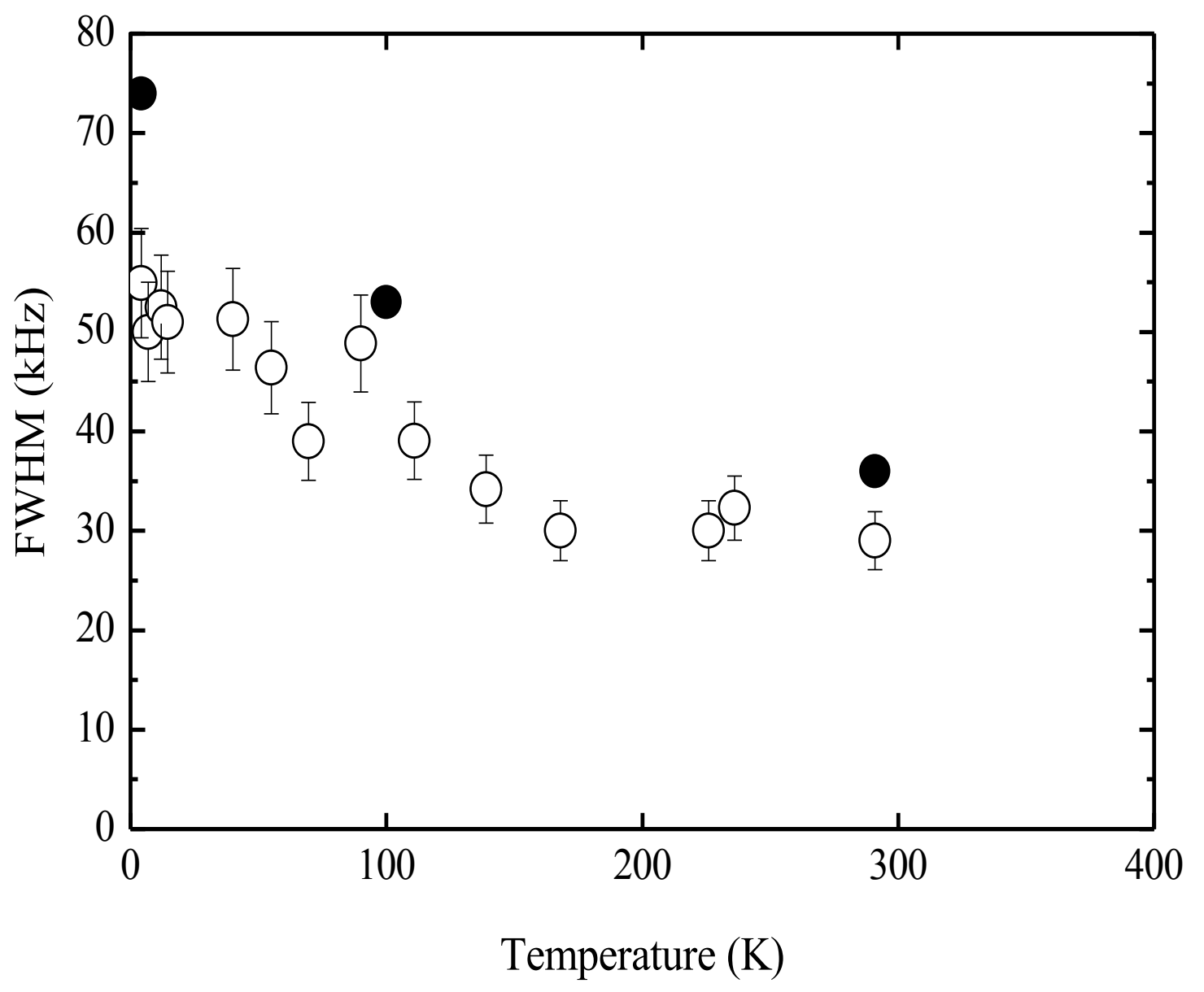

Fig 3 Procissi et al Phys Rev B 


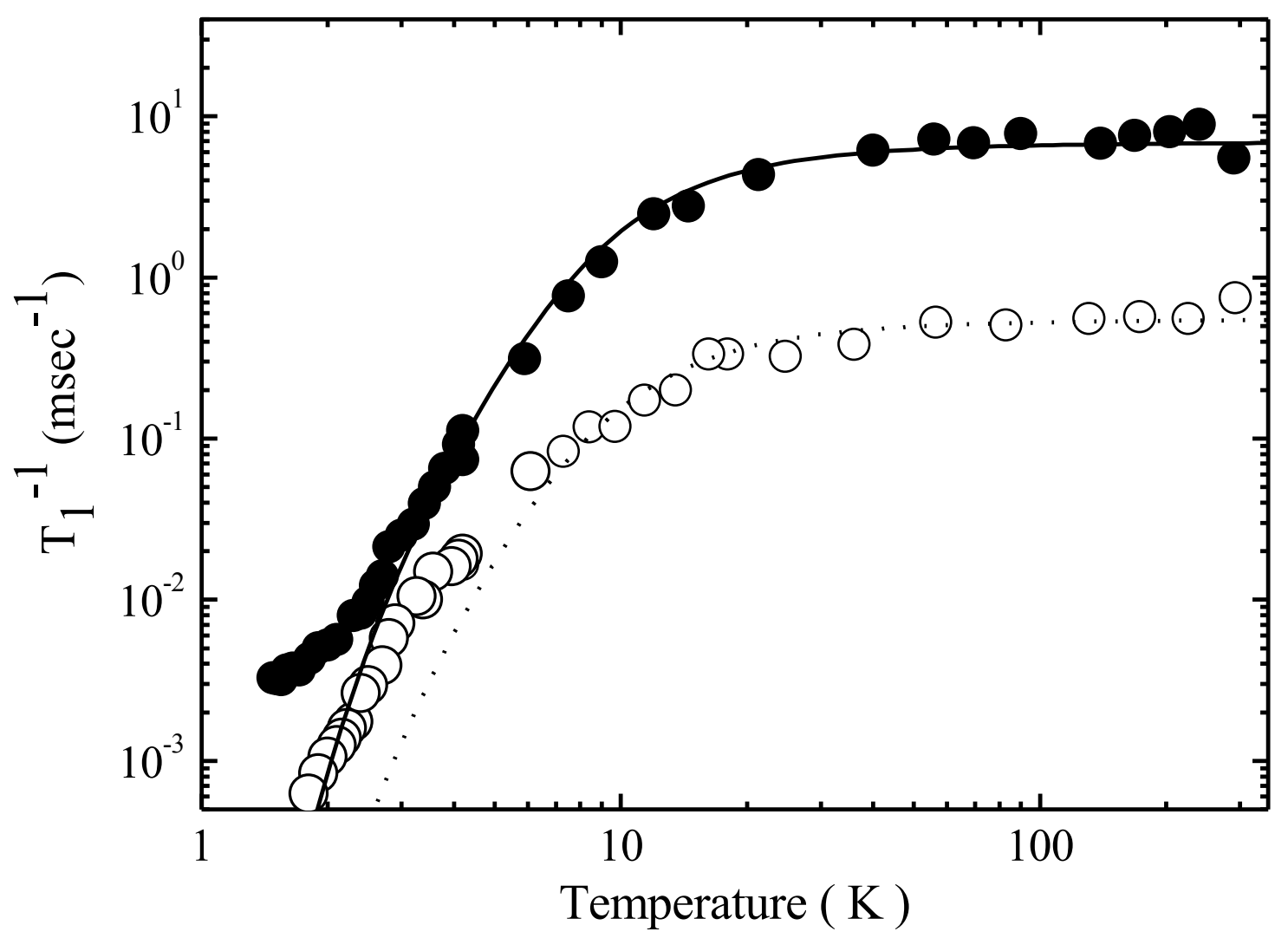

Fig 4 Procissi et al Phys Rev B 

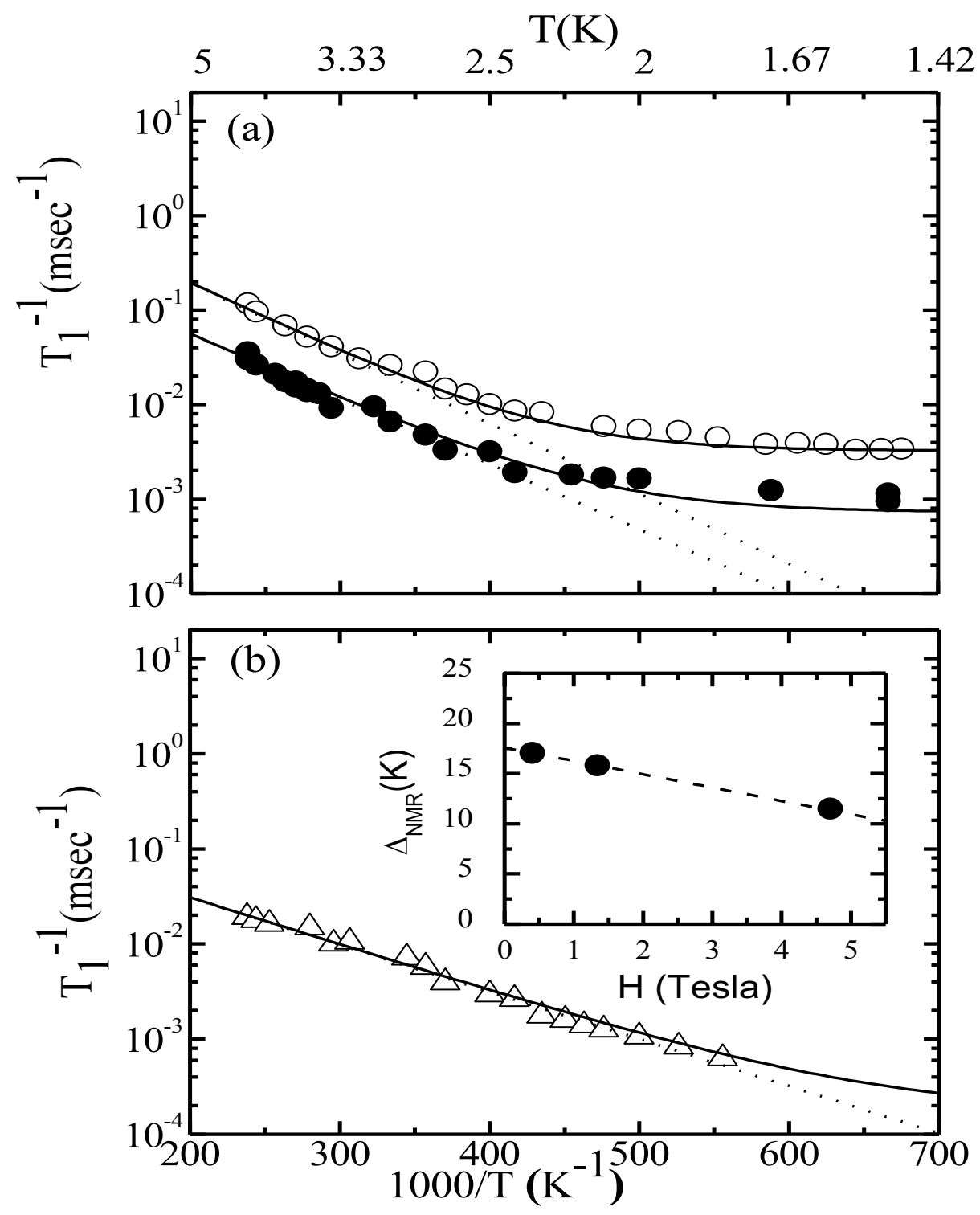

Fig 5 Procissi et al Phys Rev B 


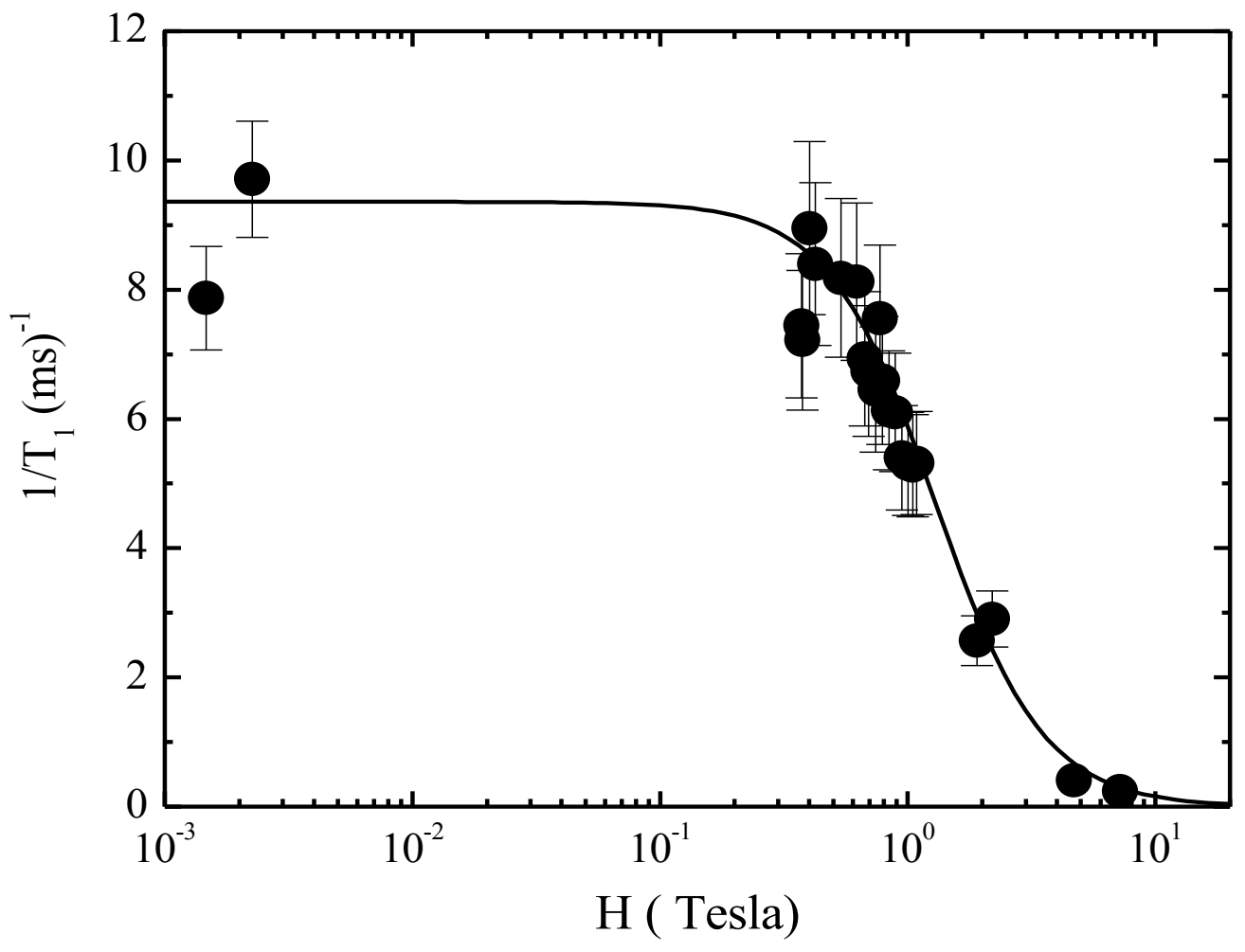

Fig 6 Procissi et al Phys Rev B 

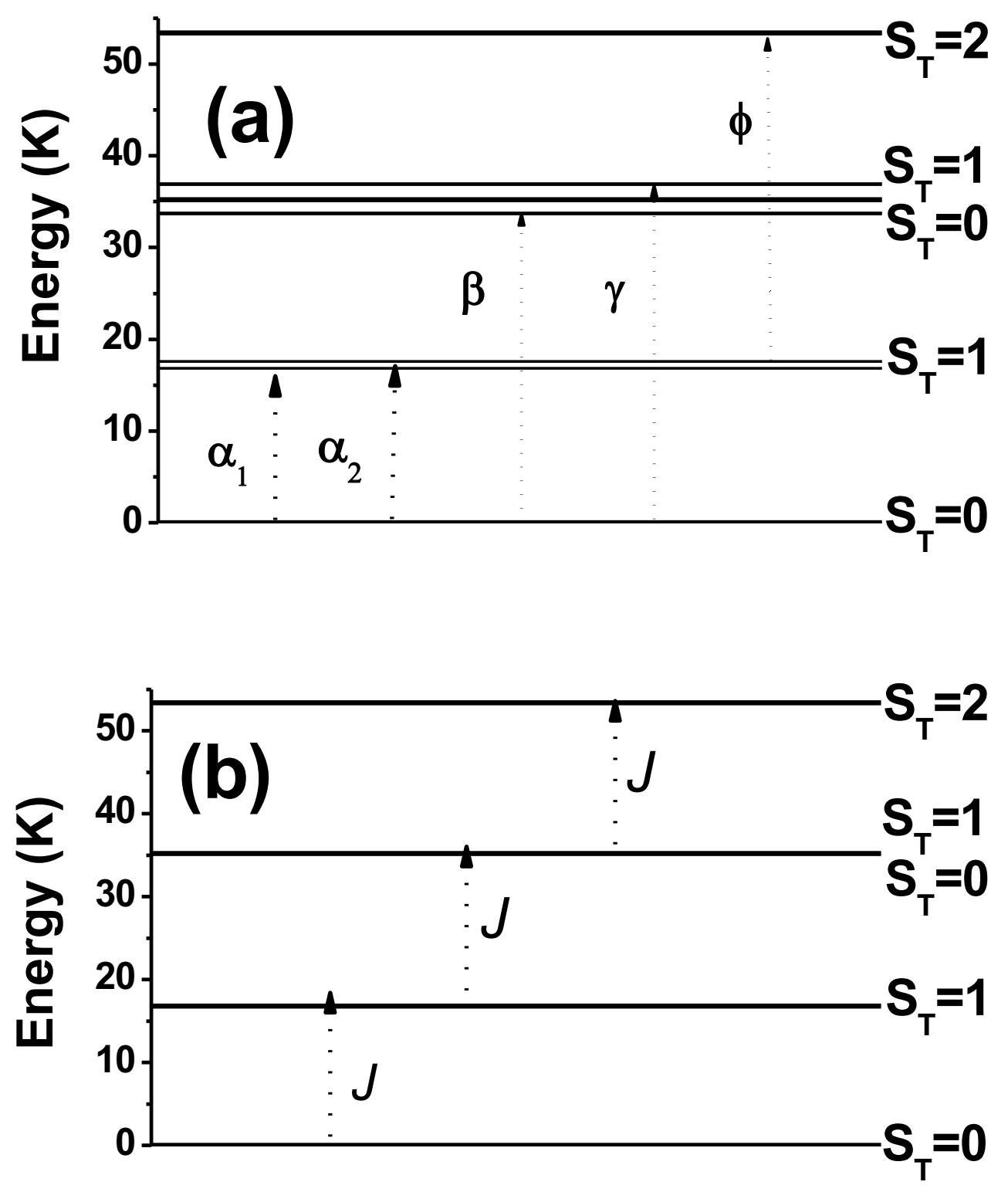

Fig 7 Procissi et al Phys Rev B 
